\title{
Risk factors of latent tuberculosis infection and immune function in health care workers in Suzhou, China
}

\author{
Cui-Lin Shi ${ }^{1 \#}$, Jun-Chi Xü ${ }^{1 \#}$, Hui Chen ${ }^{1}$, Zhi-Jian Ye ${ }^{1}$, Xin-Nian Chen ${ }^{1}$, Pei-Jun Tang ${ }^{1}$, Li-Ling Ma ${ }^{1}$, \\ Zai-Xiang Tang ${ }^{2}$, Mei-Ying $\mathrm{Wu}^{1}$, Ping $\mathrm{Xu}^{1}$
}

${ }^{1}$ Key Laboratory of Tuberculosis Prevention and Therapy, The Fifth People's Hospital of Suzhou, Suzhou 215000, China; ${ }^{2}$ Department of Biostatistics, School of Public Health, Medical College of Soochow University, Suzhou 215123, China

Contributions: (I) Conception and design: MY Wu, P Xu; (II) Administrative support: MY Wu, P Xu; (III) Provision of study materials or patients: CL Shi, JC Xu, H Chen, ZJ Ye, XN Chen, PJ Tang, LL Ma; (IV) Collection and assembly of data: CL Shi, JC Xu; (V) Data analysis and interpretation: CL Shi, JC Xu, ZX Tang; (VI) Manuscript writing: All authors; (VII) Final approval of manuscript: All authors.

\#These authors contributed equally to this work.

Correspondence to: Mei-Ying Wu; Ping Xu. The Fifth People's Hospital of Suzhou, 10, Guangqian Road, Suzhou 215000, China.

Email: wu_my@126.com; xuping19670822@126.com.

\begin{abstract}
Background: The Chinese government has pay attention about tuberculosis infection among medical staff in infectious disease hospitals, but the effects have not yet been reported. This study will explore latent infection and immune function in the medical staff and systematically analyze the associated influencing factors.

Methods: Ninety-four medical staffs were enrolled and 20 medical staffs were defined as low risk group and others were high risk group. We used IFN- $\gamma$ release assay and flow cytometry to analyze the latent TB infection status and immune function. Logistic regression analyses were performed to identify the independent risk factors of latent TB infection.

Results: This study explored and compared the infection status of medical workers and found that the rate of positive TB-IGRA results was higher among high risk group than in low risk group. Working environment, occupational history and work type were risk factors for TB infection in hospital. This study also found that high risk group had higher IFN- $\gamma$ expression and a lower ratio of CD4+ to CD8+ T cells and further analysis found that this immune disorder is associated with wards and occupations.

Conclusions: This study through rigorous sample collection and analysis found the risk factors of latent tuberculosis infection in health care workers. This finding may provide a theoretical basis to be used by the countries with a high TB burden to further improve their strategies for the prevention of TB infections in hospitals and may give an indication for improving the personal health of medical staff in infectious disease hospitals.
\end{abstract}

Keywords: Medical staff; tuberculosis; immune function; hospital infection

Submitted Jul 12, 2018. Accepted for publication Oct 24, 2018.

doi: $10.21037 /$ jtd.2018.10.112

View this article at: http://dx.doi.org/10.21037/jtd.2018.10.112

\section{Introduction}

Tuberculosis (TB) has existed for millennia and remains a major global health problem. In 2016, 6.3 million new cases of TB were reported (up from 6.1 million in 2015) (1). The medical staffs in medical institutions that treat TB who come in contact with many patients or are directly exposed to samples containing TB are at a high risk of biological contamination and occupational exposure (2). The risk of TB transmission is higher among patients and medical staff and in the laboratory than among the general population. Some studies suggest that general medical staff and staff 


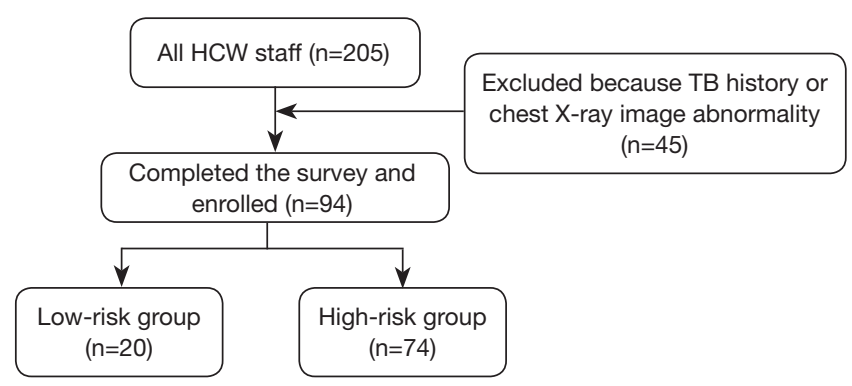

Figure 1 Study flow diagram. Of $114 \mathrm{HCW}$ s answered the questionnaire and agreed to be tested for LTBI. HCWs, health care workers; LTBI, latent tuberculosis infection; CT, computed tomography; TB, tuberculosis.

involved in TB prevention and control are in a high-risk environment and clearly have higher rates of $\mathrm{TB}$ infection and TB incidence than does the general population (3-6). Medical staff who participate in TB-related work for more than 1 year have a relative risk of infection 1.5-2.4 times the risk of other groups (7). In high-income countries such as the United States, Canada, and France (2), which implement early TB infection control measures including special TB clinics and triage systems, negative pressure isolation wards and mechanical ventilation devices, and medical respirators or masks for medical staff, etc., the infection rate of TB is between $1.2 \%$ and $50 \%$, and the incidence of TB is 130 per 10,000 in Portugal. However, in low- and middle-income countries such as Brazil (8), India, Vietnam (9) and Uganda (10), etc., the infection rate of TB is between $41 \%$ and $72 \%$, and the incidence of TB is between 558 and 600 per 10,000 (7). The conditions of implementation of TB infection control measures vary. High-income countries implemented standard infection control measures earlier; however, although low- and middle-income countries have also implemented TB infection control measures to a certain extent, the measures lack normalization and comprehensiveness $(5,11,12)$. The World Health Organization launched new "TB infection control guidelines" in 2009, and has called on countries to make plans, to regularly perform TB screening of medical staff, and to include in the record the date and results of screenings. China has a large number of TB cases; additionally, half of these new cases are infectious. Therefore, this important and urgent public health problem necessitates analysis of the current TB infection status of Chinese medical staff, improvement of the understanding of TB among these workers, and implementation of the required measures to strengthen $\mathrm{TB}$ understanding and prevention. In 2010 and 2011, China conducted related studies on the TB infection status of medical staff in specialized TB hospitals $(13,14)$. However, China has allocated a large amount of manpower and financial resources to TB prevention and control in the past 5 years, and the protection and control measures in specialized TBs hospitals have been greatly improved. There have been no studies on tuberculosis hospital infections in the past five years, and the immune status of tuberculosis medical staff has not been studied also. Therefore, it is important to reanalyze the TB infection conditions of medical staff in specialized TB hospitals to provide effective methods for other high-burden countries to better prevent TB infection in hospitals.

\section{Methods}

The study was approved by the Research Ethics Committees of the Fifth People's Hospital of Suzhou (No. 2017003), informed consent was respectively obtained from all participants before their enrollment in this study and all methods were performed in accordance with relevant guidelines and regulations.

\section{Subjects}

The epidemiological method of cluster sampling was adopted in this study (Figure 1); all medical staff members $(n=205)$ in our specialized TB hospital were chosen as the experimental group; 139 completed the questionnaire (Figure 2) but 45 were excluded because TB history or chest $\mathrm{X}$-ray image abnormality; the 94 medical staff were enrolled finally.

Exclusion criteria applied to staff who could not participate in the physical examination because of vacation or allergies and staff who were pregnant or lactating.

All the research subjects met the following criteria: (I) no history of TB; (II) no recent symptoms or signs of active TB; and (III) no active or obsolete lesions identified by chest X-ray examination.

The research subjects were divided into the following two groups according to their degree of exposure to TB: (I) the high-risk population group (high-risk group), which included medical staff from the TB department and laboratory of the Suzhou Fifth People's Hospital; and (II) the low-risk population group (low-risk group), which 


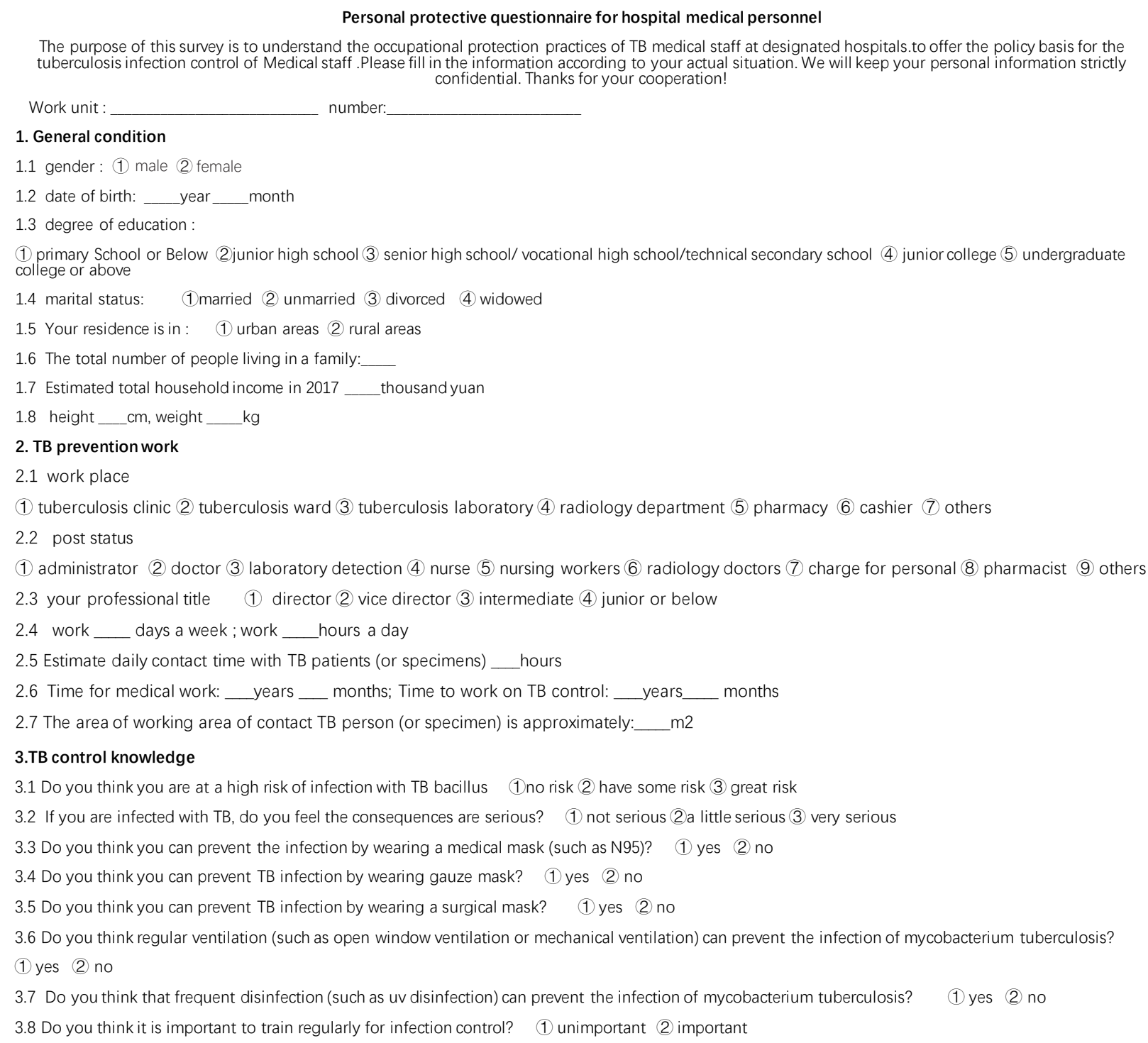

\section{Personal protective practice}

4.1 You estimate the number of TB patients (or specimens) in contact in one day: ; How many TB patients (or specimens) are exposed at most times 4.2 What mask do you wear when you are in contact with a patient?

(1) don't wear a mask (2)surgical mask (3)sand cloth mask (4) protective mask for medical use (such as N95)

4.3 The most common ventilation modes in the working area:

(1)natural ventilation (2)mechanical ventilation such as exhaust fan (3)central air-conditioning and ventilation system (4) air cleaner

4.4 The most common use of air disinfection in the working area:

(1) disinfection by ultraviolet light (2) circulating air UV air sterilizer(3) electrostatic absorption air sterilizer (4) chemical disinfection

4.5 The frequency of your work area is ventilated every day :

(1) not open (2)one to two times (3) three to five times (4) it's open all the way

4.6 Whether or not the patients you contacted always wear with a mask (at least surgical mask)

(1) don't wear (2) occasionally wear (3) most of the time (4) all the time

4.7 How often do you train for TB control?

(1) no training (2) once a year (3) once during two years (4)once during three years or above investigation date : year month day the investigator checked and signed:

Figure 2 Personal protective questionnaire for hospital medical personnel. 
was consisted of the medical staff from administrative department who did not have a chance to contact TB patients, we set this group as the general population. The high-risk group was further divided into the ward A subgroup, the ward B subgroup, the ward C subgroup and the laboratory department subgroup according to specific high-risk working environment. In addition, the highrisk group was divided into the doctor subgroup, the nurse subgroup and the laboratory staff subgroup according to profession and into the $>5$ years subgroup and the $\leq 5$ years subgroup according to occupational history. The basic conditions and infection control parameters of each group are shown in Table 1.

\section{QuantiFERON-Gold In-Tube assay}

Blood samples were obtained after fasting and before 9 AM. Three heparin tubes were collected: (I) a negative-control tube (NIL tube), (II) an antigen tube (AG tube; contained a coating of specific M. tuberculosis antigens (ESAT-6, CFP-10, TB 7.7) that came into contact with the patient's $\mathrm{T}$ cells in the blood sample), and (III) a positive-control tube containing phytohaemagglutinin-P (PHA) (MIT tube). The concentration of IFN- $\gamma$ secreted by the cells was measured by ELISA. The results were measured in IU/ $\mathrm{ml}$ and interpreted in accordance with the manufacturer's recommendations as negative, positive, or indeterminate.

\section{Flow cytometric analysis}

The fluorochrome-labeled monoclonal antibodies CD3APC, CD4-PerCP, and CD25-FITC were purchased from BD Pharmingen (San Diego, CA, USA), and IFN- $\gamma$-FITC was purchased from Cell Signaling Technology (Danvers, MA, USA). For each test, $50 \mathrm{~mL}$ of fresh heparinized whole blood of patients or healthy donors was incubated with the indicated antibodies $(10 \mathrm{~mL})$ for 15 minutes and then lysed with FACSTM lysing solution (BD Biosciences, San Jose, CA, USA). Samples were subsequently washed with phosphate buffered saline, fixed and eventually detected by a BD FACSAria supporting BD FACSDiva software.

\section{Enzyme-linked immunosorbent assay (ELISA)}

The serum samples were collected in serum separator tubes and allowed to clot for 2 hours at room temperature. Then, they were centrifuged at 3,000 rpm for 15 minutes and stored at $-80{ }^{\circ} \mathrm{C}$ for the ELISA assay. The levels of IFN- $\gamma$ in the serum were analyzed using ELISA kits (BlueGene Co., Ltd., Shanghai, China) according to the manufacturer's instructions.

\section{Statistical analysis}

The data were analyzed by GraphPad Prism 5.0 software and SPSS and were presented as the mean \pm standard error of the mean (SEM). For comparisons of the collected data, a nonparametric test (Mann-Whitney U test), Student's $t$ test or Chi-square test was performed, the bar in each group in the figures represents the mean \pm SEM. The association between risk factors and the TB-IGRA results was assessed by multinomial logistic regression, and two-tailed values of $\mathrm{P}<0.05$ were considered statistically significant.

\section{Results}

\section{Study population}

A total of 94 participants who completed the questionnaires and participated in the TB-IGRA test were enrolled in this study. These participants included 31 men and 63 women and had an average age of 33.10 years and average occupational history of 14.53 years. Among these participants, 20 subjects ( 15 women and 5 men) were in the low-risk group, with an average age of 33.05 years, whereas 74 subjects ( 53 women and 21 men) were in the high-risk group, with an average age of 37 years; there were no statistically significant differences between the two groups regarding gender or age. The high-risk group was further divided into four wards: 23 cases in the ward A subgroup, 17 cases in the ward B subgroup, 18 cases in the ward C subgroup and 16 cases in the laboratory subgroup. When divided according to profession, the high-risk group was divided into the doctor subgroup ( $\mathrm{n}=23$ cases), the nurse subgroup ( $\mathrm{n}=32$ cases), and the laboratory subgroup ( $\mathrm{n}=19$ cases). The high-risk group was also divided into the $>5$ years subgroup $(n=43)$ and $<5$ years subgroup $(n=31)$ according to occupational history. Finally, the high-risk group was divided into the lower-value subgroup $(\mathrm{n}=37)$ and the high-value subgroup $(\mathrm{n}=37)$ according to peripheral IFN- $\gamma$ levels (Table 1).

\section{TB-IGRA test results}

Two cases in the low-risk group had positive TB-IGRA results, resulting in a positive rate of $10 \%$, whereas 32 


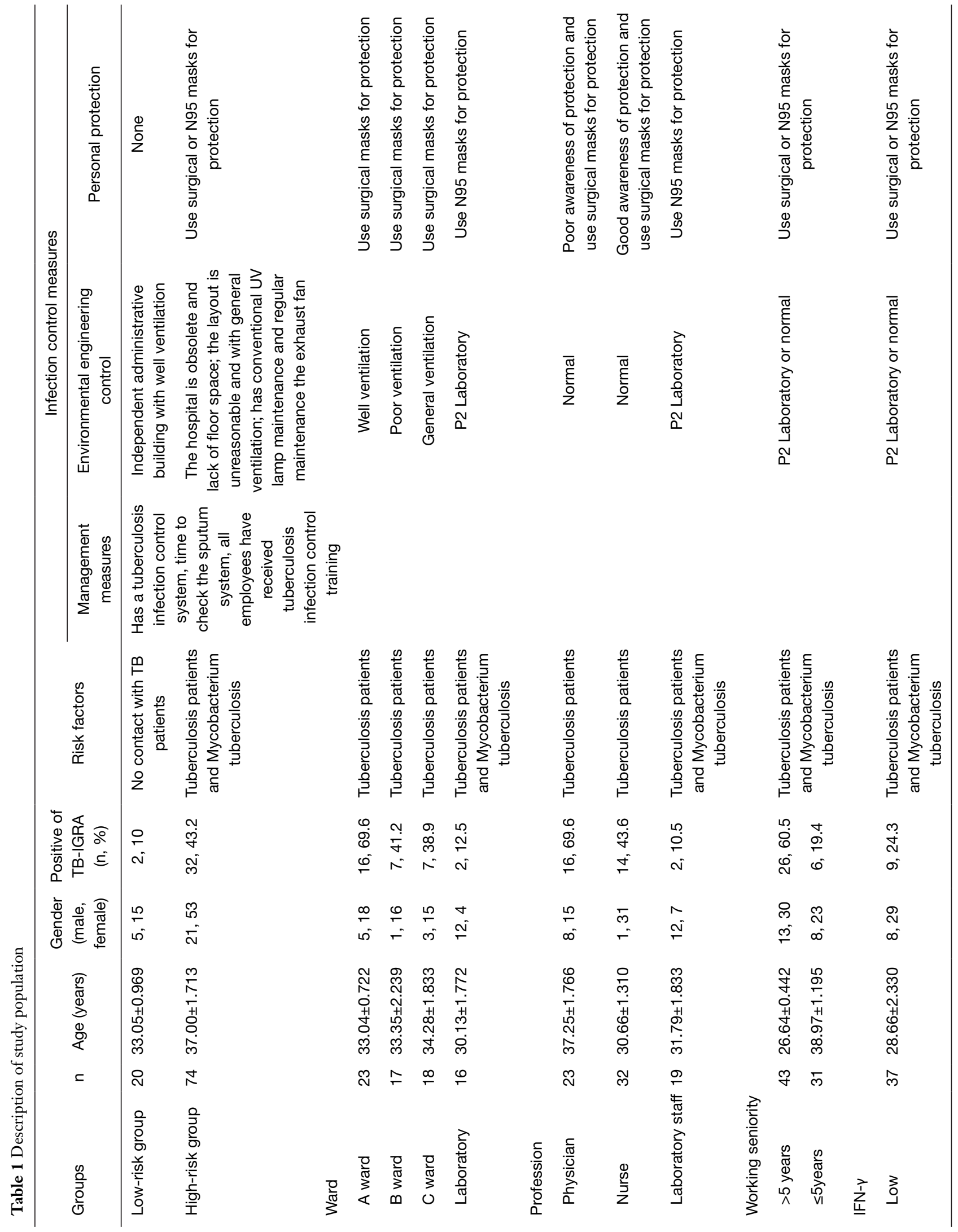


Table 2 Univariate analysis (Chi-square test, Mann-Whitney test) of the differences between the TB-IGRA positive and negative in sex, working seniority, profession, ward and IFN- $\gamma$

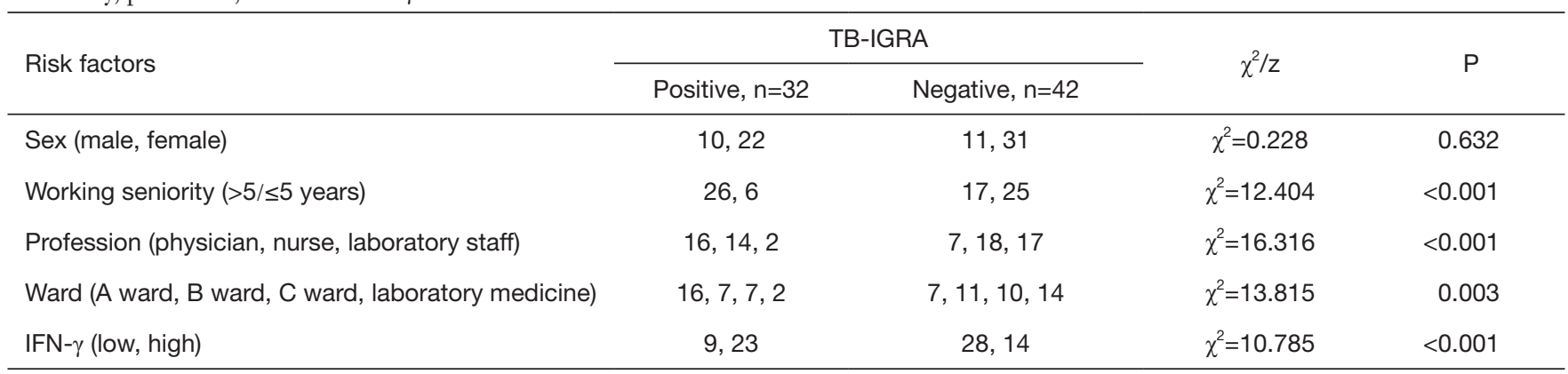

TB, tuberculosis.

Table 3 Multivariable analysis (logistic) of the differences between the TB-IGRA positive and negative in sex, working seniority, profession, ward and IFN- $\gamma$

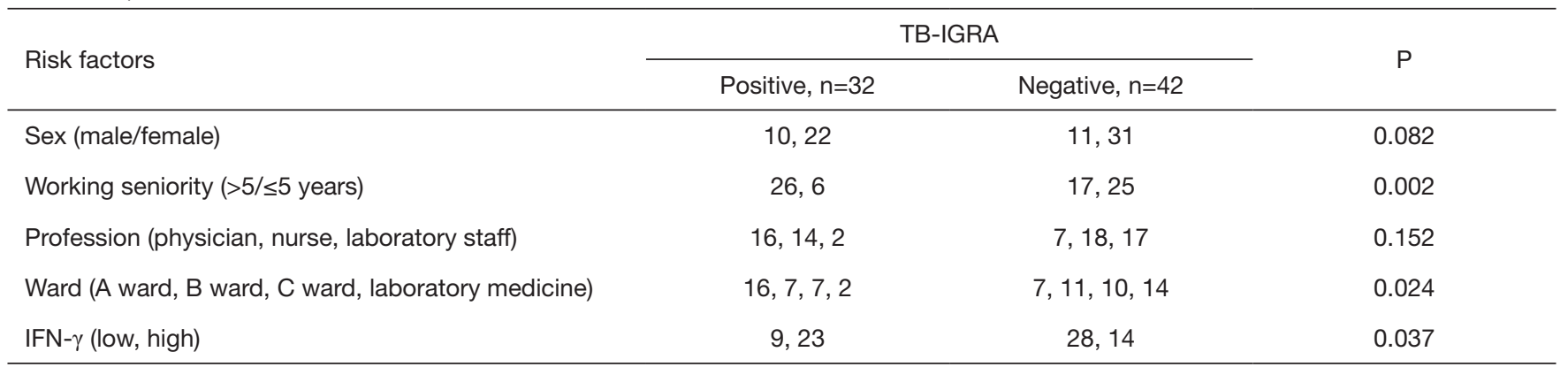

TB, tuberculosis.

cases had positive TB-IGRA results in the high-risk group, resulting in a positive rate of $43.2 \%\left(\chi^{2}=7.52, \mathrm{P}<0.01\right)$. The incidence was obviously higher among medical staff who were in close contact with TB patients than in the general population (Table 1). Subgroup analyses of the high-risk group according to profession, occupational history and work environment found that the positive rate among medical staff with an occupational history of more than 5 years was $60.5 \%$, which was significantly higher than the rate among those with an occupational history of less than 5 years $(19.4 \%)\left(\chi^{2}=12.404, \mathrm{P}<0.001\right)$, even after the logistic analysis adjustment was adjusted $(\mathrm{P}=0.002)$. The positive rate was $69.6 \%$ in the doctor subgroup, $43.6 \%$ in the nurse subgroup and $10.5 \%$ in the laboratory subgroup, and there were significant differences among these three subgroups in the single-factor analysis of variance $\left(\chi^{2}=16.316, \mathrm{P}<0.001\right)$. However, these differences disappeared after the logistic analysis was adjusted $(\mathrm{P}=0.152)$, and the positive rate was essentially the same in the laboratory subgroup and the healthy control group (10\%). The positive rate of the ward
A subgroup (69.6\%) was significantly higher than those of the other wards, while the positive rate of the laboratory subgroup (12.5\%) was significantly lower than those of other wards; the single-factor analysis of variance showed a statistically significant difference $\left(\chi^{2}=13.815, \mathrm{P}=0.003\right)$. After the logistic analysis was adjusted, there was still a statistically significant difference $(\mathrm{P}=0.024)$. In addition, the positive rate was essentially the same in the laboratory subgroup and the healthy control group (10\%). An analysis of positive values in high- and low-IFN- $\gamma$ subgroups found that the positive rate of the low-IFN- $\gamma$ subgroup (24.3\%) was significantly lower than that of the high-IFN- $\gamma$ group $(62.1 \%)$, and both the single-factor analysis of variance $\left(\chi^{2}=10.785, \mathrm{P}<0.001\right)$ and the logistic analysis showed statistically significant differences $(\mathrm{P}=0.037)$ (Tables 1-3).

\section{Correlations between the levels of serum albumin, IFN- $\gamma$,} CD4+ T cells, CD8+T cells and TB infection

Analysis of the correlation between risk of exposure/ 
infection and immune function found that medical staff in the high-risk group had higher IFN- $\gamma$ levels, a higher proportion of CD4+ T cells, and a lower proportion of $\mathrm{CD} 8+\mathrm{T}$ cells than the low-risk group (all $\mathrm{P}<0.05$ ), while the other comparisons did not identify any differences (Figure 3).

Further analysis of the differences between the positive TB-IGRA subgroup and negative TB-IGRA subgroup within the high-risk group found statistically higher IFN- $\gamma$ values in the positive TB-IGRA subgroup compared with those in the negative TB-IGRA subgroup $(\mathrm{P}<0.05)$; however, no difference was found in the other comparisons (Figure 3).

\section{Correlations of levels of serum albumin, IFN- $\gamma, \mathrm{CD} 4+$ $T$ cells, and CD8+T cells with occupation, working environment and occupational history}

The correlation analysis of occupation and immune function found statistically higher IFN- $\gamma$ values in the nurse subgroup $(\mathrm{P}<0.05)$ (Figure 4). The correlation analysis of working environment and immune function found that IFN- $\gamma$ levels were the highest in the ward $\mathrm{C}$ subgroup compared with those of the ward A subgroup $(\mathrm{P}<0.01)$, ward B subgroup $(\mathrm{P}<0.001)$ and laboratory subgroup $(\mathrm{P}<0.001)$, with all differences being statistically significant (Figure 4). Additionally, the proportion of CD4+ T cells was higher in the ward $\mathrm{C}$ subgroup than in the laboratory subgroup, and the CD4+ $\mathrm{T}$ cell proportion of the ward A subgroup was lower than those of the ward $\mathrm{B}(\mathrm{P}<0.01)$ and ward $\mathrm{C}(\mathrm{P}<0.01)$ subgroups (Figure 4$)$. No other differences were found.

\section{Discussion}

Since 2010, the Chinese government has invested a significant amount of money into the prevention and control of TB infections in hospitals and has formulated standardized TB infection control measures by referring to the experiences of developed countries. These measures included establishing special TB clinics, establishing negative pressure isolation wards with $\geq 12$ air changes per hour (AHC), and providing N95 respirators to medical staff. In 2010 and 2011, China conducted related studies on the TB infection status of medical staff in specialized TB hospitals $(13,14)$, but these studies could not have reflected the achievements of the hospital infection control scheme that has been systematically implemented in China in recent years.
Therefore, it is important to reanalyze the TB infection status of the medical staff in specialized TB hospitals. This study is also the first to systematically discuss the immune status of medical staff in a specialized TB hospital, and the results may help to provide more effective methods to reduce TB infections in hospitals for other countries with a high TB burden.

This study compared the TB-IGRA results of medical staff members and found the results were significant improved in the past five years in China. However, this study also found that medial staff still had obviously higher positive rates than low-risk groups; these results were similar to those of the investigation by the Beijing Tuberculosis Hospital in 2011 (13) but better than the survey results in Henan (14) and Inner Mongolia (15) in 2010. Due to the particular status of Beijing in China and the different methods adopted by the Henan research, we are more inclined to select the research from Inner Mongolia as a reference, and the above results suggest that the measures adopted for the prevention of TB infection in hospitals are very efficient but require further improvement. We conducted a further analysis of whether factors related to the working environment, occupational history and type of work, were risk factors for TB infection in hospitals. Notably, infection rates of staff working in P2 laboratories and wearing N95 respirators, particularly in the study of the working environment, were significantly decreased to levels as low as those of the non-close-contact population, whereas staff wearing surgical masks had no corresponding change. This study also found huge differences in infection rates among different wards, and different ventilation conditions may be responsible for these findings based on our questionnaire analysis. This study also found that infection rates were significantly higher among doctors than individuals of other professions. The survey results demonstrated that protective consciousness was significantly lower in doctors than in the nurses and laboratory staff, which we think is the reason for the differing infection rates.

This study also systematically analyzed hospital infections and the immune function of medical staff in TB hospitals and analyzed the associated factors. Moreover, this study found that the problem of immune system disorders and health problems among the medical staff of TB hospitals should be noted by hospital administrators and medical staff.

Due to the small number of cases and single-center study, this study also has its limitation and the results 
A

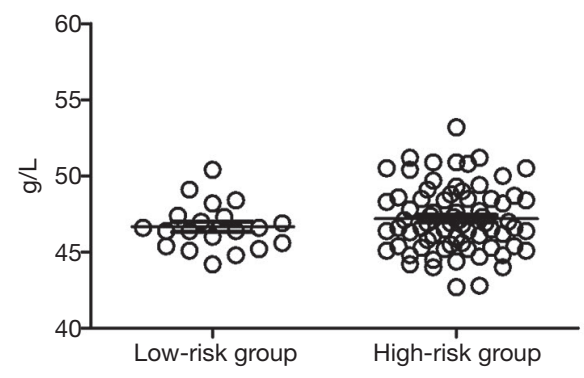

C

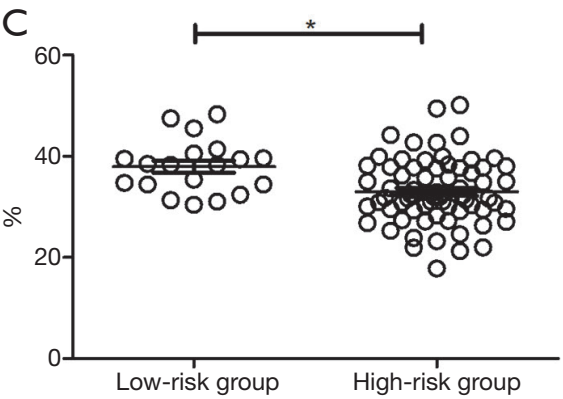

E

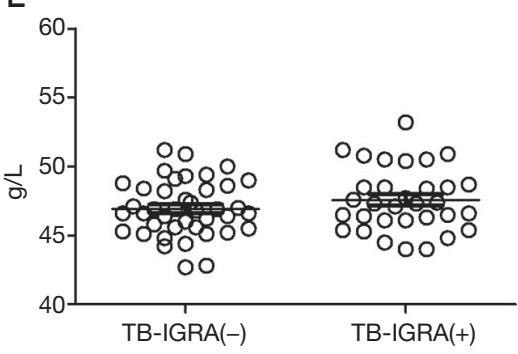

G

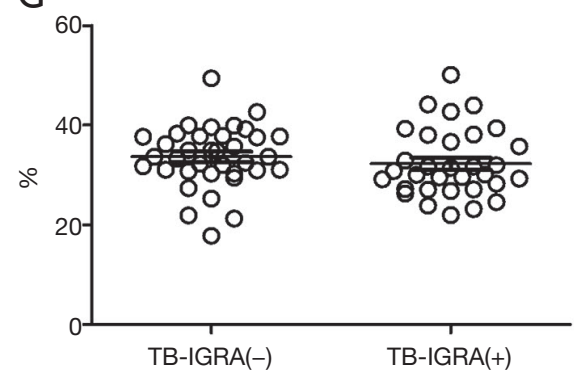

B

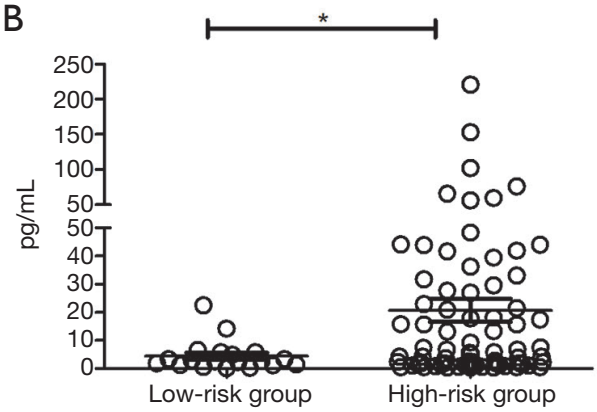

D

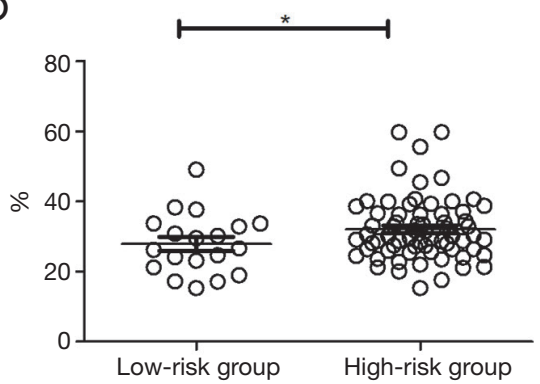

$\mathrm{F}$

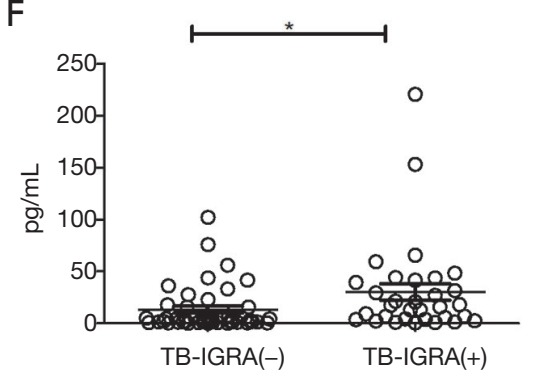

$\mathrm{H}$

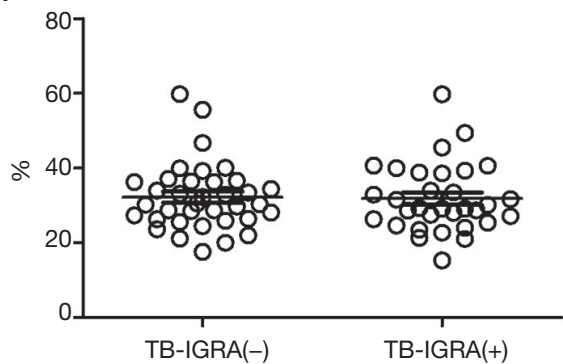

Figure 3 Analysis of the correlation between tuberculosis infection and immune function. (A) The expression levels of albumin in the low-risk and high-risk groups; (B) the expression levels of IFN- $\gamma$ in the low-risk and high-risk groups; (C) the percentages of $\mathrm{CD}^{+} \mathrm{T}$ cells in the low-risk and high-risk groups; (D) the percentages of $\mathrm{CD}^{+} \mathrm{T}$ cells in the low-risk and high-risk groups; (E) the expression levels of albumin in the TB-IGRA(-) and TB-IGRA(+) groups; (F) the expression levels of IFN- $\gamma$ in the TB-IGRA(-) and TB-IGRA(+) groups; (G) the percentages of $\mathrm{CD}^{+} \mathrm{T}$ cells in the TB-IGRA(-) and TB-IGRA(+) groups; $(\mathrm{H})$ the percentages of $\mathrm{CD} 8^{+} \mathrm{T}$ cells in the TB-IGRA(-) and TB-IGRA(+) groups. *, $\mathrm{P}<0.05$. 

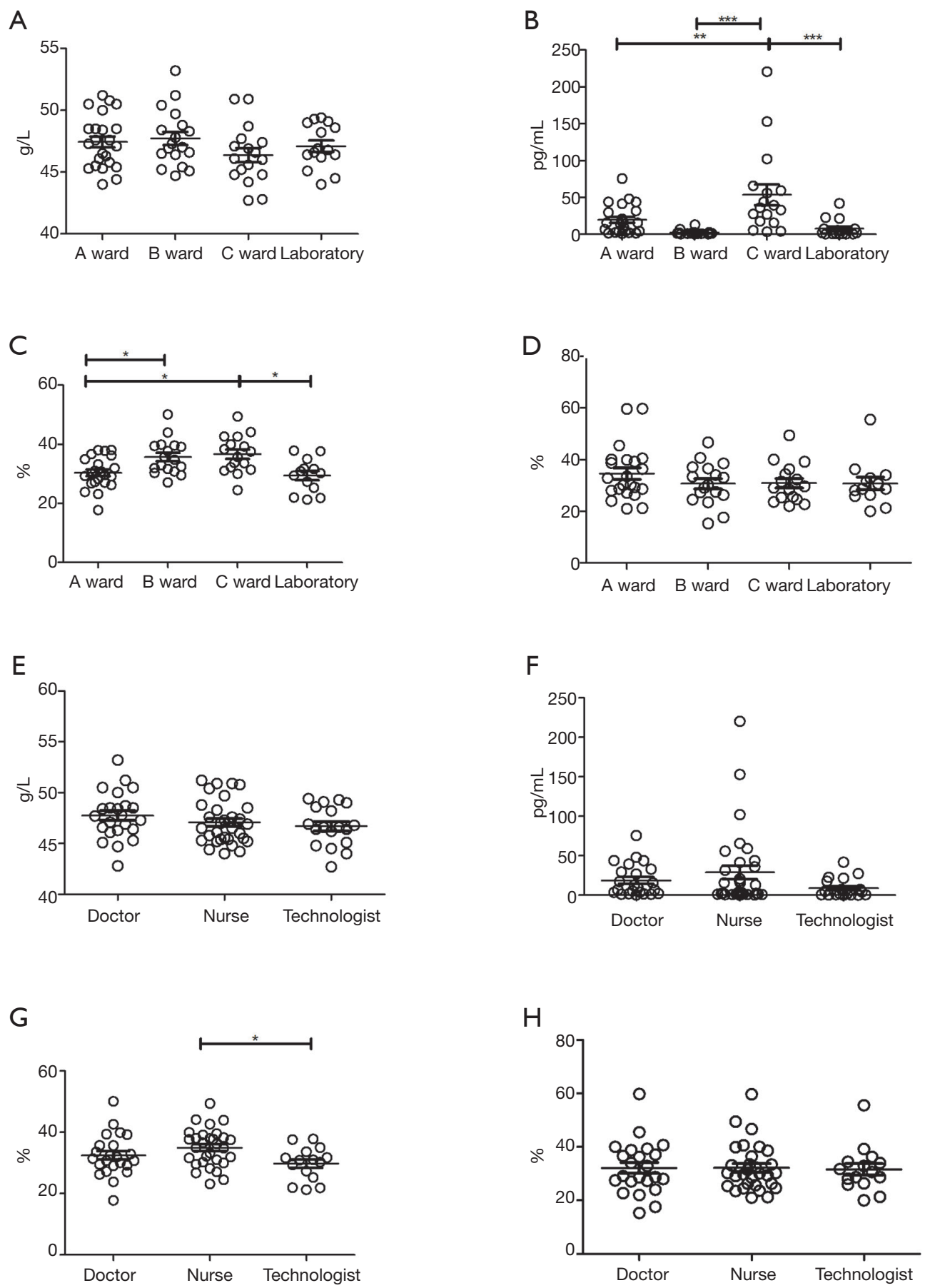

Figure 4 Abnormal immune factors among health care workers. (A) The expression levels of albumin in different wards; (B) the expression levels of IFN- $\gamma$ in different wards; (C) the percentages of $\mathrm{CD}^{+} \mathrm{T}$ cells in different wards; (D) the percentages of CD8 ${ }^{+} \mathrm{T}$ cells in different occupations; (E) the expression levels of albumin in different occupations; (F) the expression levels of IFN- $\gamma$ in different occupations; (G) the percentages of $\mathrm{CD}^{+}{ }^{+} \mathrm{T}$ cells in different occupations; $(\mathrm{H})$ the percentages of $\mathrm{CD}^{+} \mathrm{T}$ cells in different wards. * $\mathrm{P}<0.05 ;{ }^{* *}, \mathrm{P}<0.01$; ${ }^{* * *}$, $\mathrm{P}<0.001$. 
may not generalizable. So we compare the low risk group between our study and others $(2,7,13,15)$ and found there is no statistical difference. Even though, the results of this study still only consider as an indication for the prevention and possible strategies and more cases and multi-centers study still need.

This study systematically analyzed the status and factors of tuberculosis hospital infection of the medical staff and the immune charge in long-term exposure to tuberculosis. Although this study has limitations, its results still have special value. It may provide a theoretical basis for China and other countries with a high TB burden to further improve their strategies regarding the prevention of $\mathrm{TB}$ infection in hospitals and may give an indication for the improvement of the personal health of medical staff in infectious disease hospitals.

\section{Acknowledgements}

We thank all medical staff and support staff who participated in this study.

Funding: This work was supported by grants from the National Natural Science Foundation of China (81573253, 81773541); the Natural Science Foundation of Jiangsu, China (BK20161230); the health plan of Jiangsu, China (QNRC2016225, H201652 and H2017068); the Science and Technology Plan of Suzhou, China (SYSD2016167 and SS201657); and the Clinical Key Disease Diagnosis and Treatment Technology Special Project of Suzhou, China (LCZX201514).

\section{Footnote}

Conflicts of Interest: The authors have no conflicts of interest to declare.

Ethical Statement: The study was approved by the Research Ethics Committees of the Fifth People's Hospital of Suzhou (No. 2017003), informed consent was respectively obtained from all participants before their enrollment in this study.

\section{References}

1. Organization WH. Global tuberculosis report 2017. 2017. Available online: http://www.who.int/tb/ publications/global_report/gtbr2017_main_text.pdf

2. Chai SJ, Mattingly DC, Varma JK. Protecting health care workers from tuberculosis in China: a review of policy and practice in China and the United States. Health Policy Plan 2013;28:100-9.

3. North RJ, Jung YJ. Immunity to tuberculosis. Annu Rev Immunol 2004;22:599-623.

4. Vinton P, Mihrshahi S, Johnson P, et al. Comparison of QuantiFERON-TB Gold In-Tube Test and tuberculin skin test for identification of latent Mycobacterium tuberculosis infection in healthcare staff and association between positive test results and known risk factors for infection. Infect Control Hosp Epidemiol 2009;30:215-21.

5. Schablon A, Harling M, Diel R, et al. Risk of latent TB infection in individuals employed in the healthcare sector in Germany: a multicentre prevalence study. BMC Infect Dis 2010;10:107.

6. Zwerling A, Cojocariu M, McIntosh F, et al. TB screening in Canadian health care workers using interferon-gamma release assays. PLoS One 2012;7:e43014.

7. Joshi R, Reingold AL, Menzies D, et al. Tuberculosis among health-care workers in low- and middle-income countries: a systematic review. PLoS Med 2006;3:e494.

8. Franco C, Zanetta DM. Assessing occupational exposure as risk for tuberculous infection at a teaching hospital in São Paulo, Brazil. Int J Tuberc Lung Dis 2006;10:384-9.

9. Lien LT, Hang NT, Kobayashi N, et al. Prevalence and risk factors for tuberculosis infection among hospital workers in Hanoi, Viet Nam. PLoS One 2009;4:e6798.

10. Kayanja HK, Debanne S, King C, et al. Tuberculosis infection among health care workers in Kampala, Uganda. Int J Tuberc Lung Dis 2005;9:686-8.

11. Keskiner R, Ergönül O, Demiroglu Z, et al. Risk of tuberculous infection among healthcare workers in a tertiary-care hospital in Ankara, Turkey. Infect Control Hosp Epidemiol 2004;25:1067-71.

12. Pai M, Gokhale K, Joshi R, et al. Mycobacterium tuberculosis infection in health care workers in rural India: comparison of a whole-blood interferon gamma assay with tuberculin skin testing. JAMA 2005;293:2746-55.

13. Zhang X, Jia H, Liu F, et al. Prevalence and Risk Factors for Latent Tuberculosis Infection among Health Care Workers in China: A Cross-Sectional Study. PLoS One 2013;8:e66412.

14. Zhou F, Zhang L, Gao L, et al. Latent tuberculosis infection and occupational protection among health care workers in two types of public hospitals in China. PLoS One 2014;9:e104673. 
15. He GX, Wang LX, Chai SJ, et al. Risk factors associated with tuberculosis infection among health care workers

Cite this article as: Shi $\mathrm{CL}, \mathrm{Xu} \mathrm{JC}$, Chen $\mathrm{H}$, Ye ZJ, Chen XN, Tang PJ, Ma LL, Tang ZX, Wu MY, Xu P. Risk factors of latent tuberculosis infection and immune function in health care workers in Suzhou, China. J Thorac Dis 2018;10(12):67426752. doi: $10.21037 /$ jtd.2018.10.112 in Inner Mongolia, China. Int J Tuberc Lung Dis 2012;16:1485-91. 